Journal of Computer Science 2 (3): 269-275, 2006

ISSN 1549-3636

(C) 2006 Science Publications

\title{
Supporting Tools to Simplify the Navigation Process over the Web
}

\author{
${ }^{1}$ Chabane Khentout, ${ }^{2}$ Mahieddine Djoudi, ${ }^{1}$ Lamri Douidi \\ ${ }^{1}$ Department of Computer Science, University Ferhat Abbas of Setif, Algéria \\ ${ }^{2}$ Laboratoire SIC et Equipe ERTe IRMA \\ University of Poitiers Bât. SP2MI, Téléport 2, Bvd Marie et Pierre Curie \\ BP 3017986962 Futuroscope Chasseneuil Cedex \\ FRANCE
}

\begin{abstract}
We Navigation through the Web has problems of positioning and space-temporal reference marks. Therefore, it's necessary to design some computer-aided tools to help the user to position himself in space and time during a navigation session. The user is provided with a map of visited sites, thus giving an explicit representation of virtual space. Various levels of visualization are set up to make the map more visible and less overloaded. A permanently displayed time indicator enables users to keep an eye on themselves and thus to optimize navigation time.
\end{abstract}

Key words: disorientation, cognitive overload, navigation helper, navigation maps, time indicator

\section{INTRODUCTION}

The information and communication technologies, on which new training and learning media are based, have improved the transmission and access of data. But they did not facilitate enough the interaction between the user and the information. This interaction is very important factor for someone who is trying to learn. Until now the new Internet technologies are taught of to improve the speed of access and the quantity of information accessed. There is a need to develop tools to help the user interact with the huge information available on the Internet. In this context we propose a set of computer-aided tools to ease the process of interaction and understanding of the user who is absorbed by the web.

\section{OBJECTIVES AND APPROACH}

In a previous work ${ }^{[1]}$ we conducted a survey to build an educated point of view among the general public about the use of Internet. Our objectives were to understand how people's opinion is developed and grows as they use the Internet, and measure how their opinion is affected by making the browsing simple. We are especially interested in the user interface for distance learning. We started our work with the following hypotheses.

- A negative opinion about Information and Communication Technology ICT and a resistance to their use.

- The use of ICT is very limited.

- A favorable opinion after their use.

- The simplification of the browsing mode helps to spread the experience.
The data collected concern the user opinion about education in presence or distance; problem faced during the browsing, role of helpers in places where the general public can access the Internet, and the effect of the research team members. The concerned population is made up mainly of school children and adult pursuing professional education or just looking to improve their general knowledge.

We used the following methods: direct observations, queries, interviews, and examination of products and services used or rejected. The analysis methodology is based on time management (sequential or parallel ...), space representation and the usage strategy.

\section{NAVIGATION PROCESS DIFFICULTIES}

The Web is an open, evolving, heterogeneous and nonmoderated space of information. It has problems related to any navigation in a large hypermedia system. Also it contains problems specific to choices and routing through heterogeneous information.

Problems faced while browsing the Web can be characterized as disorientation and cognitive overload [2].

Disorientation ${ }^{[3]}$ is due to the fact of loosing the link between the subject being searched for and the information shown on the screen. Disorientation is caused by the absence of reference points that the users can use as they travel through the Internet. They need to know where they came from, where they are and how to move from one place to another. Three types of problems have been observed:

- The users do not know what to do due the fact that they do not know how a hypertext system works.

Corresponding Author: Chabane Khentout, Département d'informatique, Université Ferhat Abbas, 19000 Sétif, Algérie 
- The users are unable to understand the concept of the system.

- The users have lost the navigation link.

The cognitive overload happens with a user who has only a screen to work with. This user has to know the information shown is associated with what. Many decisions have to be taken while going through a hypermedia: which link to follow, how to retrieve the ones that are of interest among the links already visited or to be visited.

The user should be able to find the information being searched while moving from one page to another by following the different links. These tasks of searching for what is needed require accessing the information in smart way. This means that we need to have the capabilities to go from one place to another, identify the document reached, evaluate it, to save it or memorize its address, and related to other documents and information.

It is very common to notice that during the use of hypermedia, the user, after few minutes of search, does not know where he really is with respect to the different notions he went through. We reach a point where we start to move from one page to another or from one site to another without gaining anything new even if some of pages and/or site may contain relevant information. This is not going to improve the knowledge of the learner $^{[4]}$.

Working with World Wide Web may lead the user, from one link to another, to a page that has very little to do with the subject being searched for. The information read, that is not related to a specific cognitive project is forgotten very quickly. Meanwhile we forget other pages that we have consulted earlier which contained information that is of interest to us. We activated a link that we taught it would allow us to get more information about the topic. This action took us further away from the subject because we kept following other links. Before we noticed it, we lost track the pages that interest us. After a half-hour of search, we turn off our computer with the impression that we went through a lot of material without learning any thing new.

\section{COMPUTERIZED ANSWERS}

The users have shown their unhappiness about Internet exploration. Especially about the time they spent and corrections of routing (links) to move from one server to another. They are aware that there is a limited knowledge of the use of computers locally. According to them, in order for this development to succeed, once the research team leaves, there must be a way to keep in touch with the experts. This point is very important, because it shows that the development of a local Internet culture depends on the capacity of the user to move from a situation where the experts are present to a situation where help is available but from a distance when needed.

This last point seems to be one of the most important things learned from this research because the use of ICT will depends on it. The experts can not be present at all times in all places. Also the move from help in presence to help from a distance which is a particular form of groupware between one or many experts and one or many users is a necessary condition for the development of ICT.

This is not limited to the use of a file or a Web site from a distance, because the notion of distance is transparent to the user. But it is extended to the case when the user realizes that he is physically alone and he needs someone to communicate with face to face to get help. Researchers in the area of distance learning have studied this problem few years ago. These researchers have shown two main factors: anxiety of loneness and the inability to organize the work ${ }^{[5]}$. But since than few more factors have emerged ${ }^{[6]}$. These mainly concern the individual abilities such as:

- The ability to identify the communication tool being used from a distance

- The ability to choose based on the situation the mode of communication, which can be synchronous or asynchronous, point to point or multi-points.

- The ability to evaluate and manage the time while using the multimedia ${ }^{[7]}$.

- The ability to describe the problem very precisely and clearly in the communication language. This point is very important in the development of ITC, which assumes that the users are very competent in the communication language.

We assume that these competencies are acquired by experience and/or by training. This will help to eliminate the two major handicaps mentioned above. Concerning our situation, our users consult with us very often while we are present and they will have our support from a distance when we leave their location.

Navigation Help : Navigation help can be of two different ways:

The first way is concerned with the construction of web sites. A construction method should be adapted to make it easy for the user to access and search the sites. In ${ }^{[4]}$ for example, the author proposes to limit the depth decomposition of the page to four levels. This means that only three nodes can be active at the same time. In addition, each screen should have about five active links. In order to be clear and efficient links to general ideas of dependant information are favored. This approach of construction will result into hypermedia with a simple structure, which is more efficient. The inconvenience of this method is, the user has to split for example a design of complete course into subsections which are accessed separately. But we can always links these subsections to each other indirectly. 
The second way is to provide a set of computer aided tools that will allow the client user to navigate the web with ease using his/her preferred browser. The general browsers, Netscape or Internet Explorer propose some functionality such as history, and bookmarks but these kinds of help are insufficient for the user needs. In addition, the users of a hypertext system create different representations.

Many computer-aided systems to help the users to browse the Internet have been proposed in the literature. Among them are Nestor [8], MAWA [9] Broadway ${ }^{[10]}$, FootPrints ${ }^{[11]}$, Hypercase ${ }^{[12]}$, and Letizia [13]. A comparative study of some of these tools is available in ${ }^{[7]}$. Nestor and Broadway are the closest to our design of computer aided tools to navigate the Internet.

NESTOR browser is developed by CNRS-GATE laboratory and it is similar to Netscape or Internet explorer. It runs under Windows 95,98 or NT on personnel computer. NESTOR main screen is divided into two windows. In the right window a classical browser based on the component Active $\mathrm{X}$ Internet Explorer is displayed. A graphical and interactive help window is displayed on the left. A map is drawn automatically as the user browses the Internet. The user can edit this map, and can use it to go directly to a site that he visited before. This navigator is built to achieve the following two main goals: help the trainee to become an active leaner and make the browsing easy because most of the users have little experience with Internet. It is important to help them make full use of their experience ${ }^{[8]}$. NESTOR is a complete and excellent navigator. It is a very good tool to build the navigation map. However, NESTOR is plate form dependent, it works only under Window with Internet Explorer. Also it does not keep track of the time factor.

Broadway is a computer-aided tool for navigation of the web that uses case reasoning to recommend pages for visit according to the behavior of the current user. Broadway can be accessed by a group of users and supports indirect cooperation. The system keeps track of four parameters related the users' navigation. These parameters describe the address, the content, the explicit evaluation and the ratio of display of each web page visited. Thy will be the base to extract useful cases to be used in the future. The evaluation of a sequence of pages gives an indication of the behavior of the user being observed. The index model used allows the modeling of these types of cases. Broadway is extended with a new tool that keeps track of the user behavior in a large number of variables. A detailed and flexible behavior management is possible due to the extensive observation combined with the indexing model ${ }^{[2]}$. Broadway does not include the navigation time as a user parameter. But it remains a very good tool to model the user behavior during a browsing session.

\section{DESCRIPTION OF THE SYSTEM}

In order to allow the user to keep track of time and to know where he/she is, we have designed and implemented a computer-aided system for virtual navigation of the web called NaVir ${ }^{[14]}$. This system which is implemented in Java can be used with any browser (Netscape, Internet Explorer or other). The main screen is made up of many windows. Its kernel is made up of two important modules: one is to collect the different URL addresses and the other is to build and interact with the graphical map and the management of navigation time ${ }^{[15]}$. The user has access to a dictionary containing the frequently used words in Internet that may not be understood. Also help for the system can displayed in a separate window.

\section{SOFTWARE ARCHITECTURE}

In order to guarantee that our system is independent of the browser, the way we recuperate the addresses of the sites/pages visited is using a proxy server. This proxy server seats in between web clients and information servers using different protocols. It is used to pass the information from one end to the other. Each user's request is sent by the client to the proxy server which will respond directly if it has the information in its cache, or it will pass the request to the destination server. The proxy server keeps a copy of each document it sends in its cache. This copy is kept for variable amount of time. This way if a document is requested and is available in the cache of the proxy there is no need to get it from the destination server. The management of the cache is done based on the following parameters: date of the last time when the document was updated, maximum time that a document can spend in the cache and for how long has the document been in the cache without being used. This service which is transparent to the user, makes the responses to the user requests more efficient. It also reduces the traffic on the network.

The proxy server receives the requests from the browser, rearrange them if needed and sent them to the module that is responsible to build the map. This server is installed locally on the user's machine to serve as a link for HTTP requests. The browser has to be configured to use this proxy server. Each HTTP request will be intercepted and sent by the proxy after extracting the necessary information (address requested, elapsed time since the last time this address was requested) and saves it. This data is stored in a file that will be used by the module responsible for building the map later on. 


\section{GRAPHICAL MAP FOR NAVIGATION}

The development of a graphical map and its use as a computer-aided tool for web browsing is based on the studies of cognitive processes that happen during the navigation of distributed hypermedia. It is a graphical representation at the same time of conceptual and geographical search path followed by a user while searching for a particular topic. The Navigation map that we designed is based on the idea used in conceptual maps ${ }^{[10]}$.

A conceptual map is a new way of representing the relationship between a set of knowledge and the nature of this relationship. It is a graphical representation of links among different concepts about the same topic. It should evolve with the knowledge of the trainee.

The conceptual map is also a computer-aided tool for navigation. It allows a hypertext reader to see on the screen the titles of information units and the links that connect them in a form of a network. It is drawn with a goal in mind, within well-defined references, and according to a graphical representation suitable for browsing problem.

\section{CLASSIFICATION OF GRAPHICAL REPRESENTATIONS}

Browsing the Web implies the manipulation of huge amount of information. The major role of the graphical interface of system developed for this purpose is to make this information easy to comprehend by the users. This is based mainly on the graphical representation of the different pieces of information and the relations connecting these pieces together. The graphical interface between the users and the system is a way to construct the image of the system. A review of the literature indicates the existence of many graphical representations. So it is necessary to study and classify these different representations.

The taxonomy developed in ${ }^{[16]}$ is based on the notion of the user's actions. The classification proposed emphasize the nature of actions (direct or indirect selections), their levels (single, group, and attributes and objects integrity) and their effect on the graph, on the representation and the transformation or organization of the objects selected.

The study proposed by [17] classifies representation techniques in five categories: geometric, network based, hierarchy, pixel oriented, and iconic. This approach has the disadvantage of mixing construction and graphical tools used as a classification creteria, which makes it very difficult to characterize some systems.

The approach described in ${ }^{[18]}$ is based on the type of data represented and the low level task performed by the user on this data. The author then listed different graphical representations used for each type of data. He also identifies seven task types that the graphical representation should favor. The high level tasks that are independent of the data being manipulated are: general view of the information, zooming, filtering, getting the details, link representation together, having a history of actions performed, and extracting part of the information so that it can be used by other applications. Three of these points (general view of the information, zooming, and getting the details) are considered during the conception of the representation. IN $^{[19]}$, the authors propose to characterize the graphical representation based on a chosen point of view about the data but not on the type of data. A point of view is defined by deciding what is necessary out of the data that should be given to the users based on his needs to perform his task in a satisfactory manner. If we are unable to characterize in a precise way the object's activities then the graphical representation should be flexible enough to detect one or many points of view that are suitable to accomplish the task. For a set of data we might have more than one point of view depending on how the data is considered. These points of views might complement each other for the purpose of the user's activities. So it necessary to be able to represent simultaneously many views which means we should choose a graphical representation guided by multiple points of view. This corresponds to multiple views discussed in ${ }^{[20]}$ and ${ }^{[21]}$. This multiplicity should be taken as a factor during the design of an interface that can adapt itself to different tasks.

\section{CHOICE OF GRAPHICAL REPRESENTATION}

The navigation map gives the possibility to keep track of path followed by the user while browsing the web. The map is modeled by a directed graph. Each page address (URL), the topic or title of the page, and the time spent connected to this page are kept in the nodes. The map is displayed upon request of the user at any stage of the browsing.

A directed graph representation of the map is most suitable for its visualization. Each node contains the name and the information of the page visited. The information kept should be in such way that it does not affect the clarity of the graph. The nodes are connected to each other to indicate the fact that the user has moved from a specific page to another. The nodes should be displayed on the screen in a way that all are visible and with minimum edges intersection.

To choose the best representation of the map, we looked into different techniques (available in the literature) to display graphs. Also we kept in mind the specific properties of our graph and the different operation that are performed on it. We found that the circular graph representation is the most suitable for our case. In this approach, all the nodes are drawn on a circle. The information represented in the graph is very 
easy to read. The nodes do not touch each other, and are uniformly distributed over the circle. The edges may cross one another, but the intersection is concentrated around the center of the circle. It is very clear which edges are coming or going out of a node. The user can modify this representation as it is explained later.

\section{MANIPULATION OF THE MAP}

In addition to the automatic graphical map generation representing the visited pages, the system allows the drawing of the map from a list of identifiers of preselected pages. Also the user can follow the map evolution by creation, deletion of any link or reorganization of the graph, or do only a read of the map for a simple task. All actions that are performed and amount time spent on each page are saved and used for evaluation. This information can be shared among a group of users.

The user has also the possibility to save, print or reopen the map constructed during a navigation session. He has also access to the log report during a session that will allow him to do a self-evaluation and to be able to follow his progress during a training period or a search for information. It is also possible to have report indicating the daily interactions and the time spent connected to each site. The graphical map can be used to share information within a group of learners in a cooperative learning environment. Each user can benefit from the experience of the other members of the group.

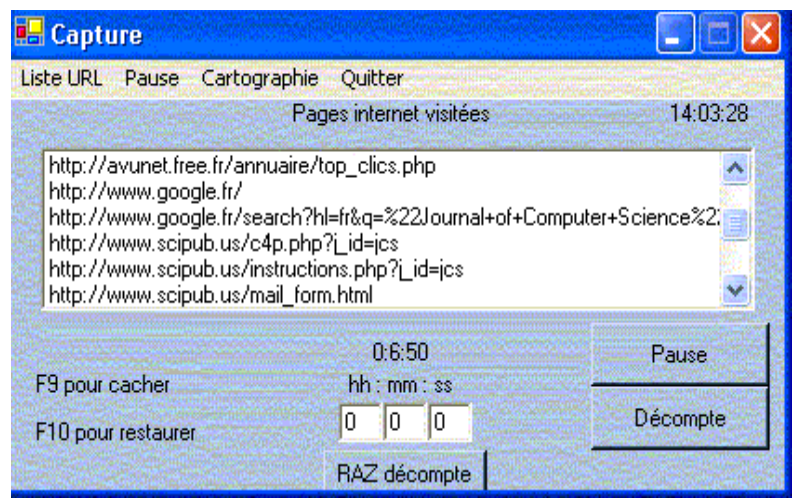

Fig. 1: Display of URL List

At any moment it is possible to go backward by clicking on "back" button. When we are in page mode, we can recognize the complete address and title of a node by simply clicking on it.

Actually to make the comprehension easy a coloring system is adapted. The node colored in green represents the first site (page if we are in page mode) visited by the user. A node colored red represents the last site visited. The intermediate sites are colored orange. If the first site visited is also the last site then it is colored in gray.

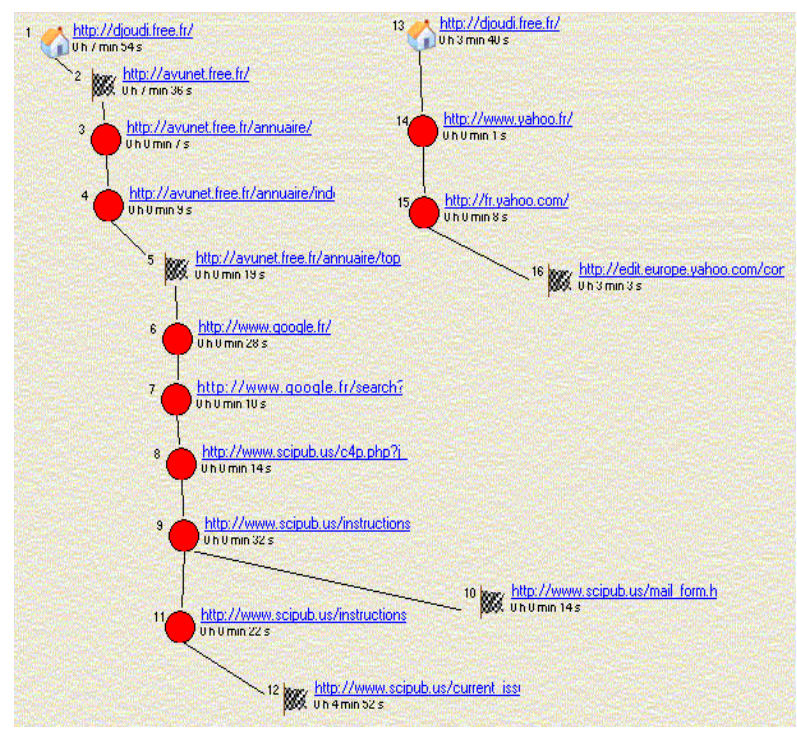

Fig. 2: Display of the Map and Time Spent

The user has also the possibility to save, print or reopen the map constructed during a navigation session. He has also access to the log report during a session that will allow him to do a self-evaluation and to be able to follow his progress during a training period or a search for information. It is also possible to have report indicating the daily interactions and the time spent connected to each site. The graphical map can be used to share information within a group of learners in a cooperative learning environment ${ }^{[26,7]}$. Each user can benefit from the experience of the other members of the group $^{[22]}$.

\section{NAVIGATION TIME}

Clock intern and clock extern: According to ${ }^{[23]}$, people do not manage the time the same way while using a media to learn or to just get information. Some users manage their time efficiently but others do not. In this regard people act in different ways. There is a huge difference in the estimation of time spent browsing from one person to another. Certainly our interest in the message being read and our motivation for browsing the Internet play a major role in our time estimation but there are other factors related to the communication tools which also influence our intuition. These observations suggest a dual notion of the time. On one hand each media has its own internal clock, on the other hand the users have their own notion of the time based on the interaction with this media.

Actually there are two kinds of speed (which imply that there are two amount of time for this execution) related to the execution of any phenomena. The first time is the universal time measured by our watches. This is the real 
time. The duration of the execution of phenomena is equal to the difference between its start and completion times. The second time is the time estimated by the user. This time does not have any mathematical base. It is related to the user's cognitive activity and his perception of the real time.

Our mathematical values loose their significance because we have a deformed perception of time. We can not define a response time in an absolute way but we define it based on the time that we perceive. While using a graphical interface, the manipulation is done in a direct way via the interface objects (keyboard, mouse, etc.) in response to these actions the represented objects change to inform the user that the event has taken place. These requirements are generally taken in consideration by the software developers. But the time dimension is rarely considered by most applications such as the available web browsers.

Time Panel : In order to give to the user the ability to be aware of the time spent during a browsing session, we added panel on which the time is displayed. When the user starts the system he will be asked to enter the expected time to be spent. Once the time expires the system will inform the user by displaying a message. Then the user can decide what to do next. The choices are continue the browsing, save the information find so far, or quit. Actually the user can request the system to display the total time spent on each node.

\section{EXPERIMENTS}

These tools presented here were in response to certain browsing problems. There are a number of computeraided tools that give the possibility to the learners to experiment. We concentrated on the problem of disorientation and putting some reference points. During the experiment, the use of usual browsers, such as Internet Explorer or Netscape, by novice users while solving a pedagogic task, is observed.

We have tested the set of tools developed in a real practical sessions in cooperation with a teacher and his students ${ }^{[17]}$. This testing was done in a course about the new information and communication technologies in general and about the web in particular. There were about 100 second year university students, aged from 19 to 22 years. Their major is earth science and all had some experience with some browser. They used the Internet to search for information before. This is a limited experience because we have only the strict minimum needed equipment. Also the connection equipment is not suitable for heavy use.

The experimental environment is made of:

Free access to the educational server (self-learning mode) as a complement to the course taken in a regular class.

A guided access to the course according to a plan, prepared by the teacher, which is made of a set of documents on the educational server and some links to public documents available on the web.

The collection of information about a particular topic from the Internet and the structure of this information into a personnel or group document will be submitted to the teacher using the browsing map.

The time panel is used with the browsing map to limit the time of search and access of information.

The use of the browsing map has given the students the possibility to structure their knowledge, recognize their limitation and to have a graphical support that can be used to prepare the plan how to search for the needed information. The proxy architecture made it possible, while using the tools, to display on the screen the browser on one window, the sequence of site and the navigation map on another. This solution helps to reduce the cognitive overload of the users.

For the teacher the graphical map can be considered as a tool to analyze the content of what is being taught, to have a better structure of the programs and manuals, and to build a plan of the course. The preparation of a guided tour with comments helps to get the new learner to start. These guided tours allow a simple browsing without limiting the freedom of exploring. They include some public documents available over the Internet and some local documents prepared for pedagogical purpose.

\section{CONCLUSION}

The computer-aided tools developed (navigation map, time, and educational server) help to solve many navigation problems according to the objectives stated earlier. But we think it is time to start solving questions related to the use and control that the user should be able to do. We plan to allow the user to add comments about each site or page visited. This is a simple way to personalize its path. The other possibility is to be able to start the browser from the graph. Clicking on a node will link the user to the corresponding address ${ }^{[24]}$.

Another goal is to evaluate the system in a cooperative learning environment. This will allow us to measure the success of our system in simplifying the browsing procedures and the search of information via the Internet.

We are currently working on improving our navigation help tool and developing other tools that are helpful to learners using the web within a platform for distance education.

\section{REFERENCES}

1. Djoudi M. and Harous S., 2001. Simplifying the Learning Process Over the Internet. Technological Horizons in Education Journal, Volume 29, Tustin, USA.

2. Souza, A. P., Dias P., 1996. Analysis of Hypermedia browsing processes in Order to Reduce Disorientation. In Proceedings of EDMEDIA'96 conference, AACE. 
3. Rhéaume J., 1997. Les hypertextes et les hypermédias. Revue éducatechnologiques, Faculté des sciences de l'éducation, Université Laval, Canada.

4. Quarteroni P., 1996. Un hypermédia pédagogiquement efficace. Revue éducatechnologiques, sous la direction de $\mathrm{J}$. Rhéaume, Université Laval, Canada.

5. Moore M. G., 1990. Contemporary issues in Americain Distance Education. New York, Pergamon Press.

6. Perriault J., 1996. Synchronous and asynchronous media in an hybrid learning process: effects of time compression and expansion. European Distance Education Network (EDEN), Proceedings of the Conference, Milton Keynes, The Open University.

7. Jaecklé L., 1998. Synchronous communication as a disturbing element of a university curriculum. Research Perspectives on Open Distance Learning, Collection of Research papers from the four projects supported by the EU Joint Action on Open Distance, Learning, Bologna, SCIENTER .

8. Zeiliger, R., Bélisle, C., Cerratto, T., 1999. Implementing a Constructivist Approach to Web Navigation Support. In proceedings of the EDMEDIA'99 Conference, AACE, Seattle, USA.

9. Singer, N., Trouillet, S., 2001.Cartographie du Web et navigation sociale : le système multi-agent MAWA. Conférence Nimes TIC: Interaction et Composition Dynamique .

10. Jaczynski M., Trousse B., 1998. WWW assisted browsing by resing pas navigations of a group of users. In Proceedings of the European Workshop of Case-base Reasoning, EWCBR'98, LNCS/AI, Dublin, Ireland, Spring-Verlag.

11. Wexelblat A., Maes P., 1997. Footprints : Visualizing Histories for Web Browsing. In Actes de la 5e conférence sur la Recherche d'Information Assistée par Ordinateur sur Internet (RIAO'97), Centre des hautes études internationales d'Informatique, Montréal, pp : 75-84.

12. Micarelli A., Sciarrone F., 1996. A Case-Based System for Adaptive Hypermedia Navigation. In Advances in Case-Based Reasoning, Proc. of the 3rd European Workshop on Case-Based Reasoning (EWCBR'96), vol 1168, pp: 266-279, Springer.

13. Lieberman H., 1995.Letizia :An Agent that Assists Web Browsing. In Proceedings of International Joint Conference on Artificial Intelligence (IJCAI'95), pages 924-929, Morgan Kaufmann.
14. Djoudi M., 1999. Navir, un système d'aide à la navigation virtuelle sur le Web. Deuxièmes Entretiens Internationaux du CNED, Poitiers.

15. Djoudi M., 2001. Conception d'assistants à la navigation sur l'internet. Dans Comprendre les usages de l'internet. d'Eric Guichard Edition ENS Rue d'ULM, ISBN 2-7288-0268-8

16. Tweedie L. A., 1995. Interactive visualization artifacts: how can abstractions inform design?. Proceedings of the CHI'95 Conference, p. 247-265.

17. Keim D. A., 1997. Visual techniques for exploring databases. In Invited Tutorial, Int. Conference on Knowledge Discovery in Databases KDD'97, Newport Beach.

18. Shneiderman B., 1998. Designing the User Interface. Addison Wesley, third edition.

19. Bruley C., Genoud P., 1998. Contribution à une taxonomie des représentations graphiques de l'information . Dixièmes journées francophones sur l'Interaction Homme Machine, IHM 98, Nantes.

20. Nigay L., Vernier F., 1998. Design method of interaction techniques for large information spaces . In Proceedings of Advanced Visual Interfaces, AVI'98, pp: 37-46.

21. Wang Baldonado M. Q., Winograd T., 1997. Sense maker: An information exploration interface supporting the contextual evolution of a user's interests. In Proceedings of ACM CHI 97 Conference on Human Factors in Computing Systems, pages 11-18.

22. Trousse B., Jaczynski M., Kanawati R., 1999. Une approche fondée sur le raisonnement à partir de cas pour l'aide à la navigation dans un hypermédia . In Proceedings of Hypertexte \& Hypermédia : Products, Tools and Methods (H2PTM'99). Paris.

23. Perriault, J., 1998. Le temps dans la construction des savoirs à l'étude des médias. Revue européenne des sciences sociales, Tome XXXVI, $\mathrm{n}^{\circ} 111$, pp : 109-118.

24. Bélisle C., Zeiliger R., Cerratto T., 1997. Integrated Cognitive Engineering at the Interface: A Tool Mediation Perspective. In Proceedings of the Second International Cognitive Technology Conference (CT'97), edited by J.P. Marsch, C.L. Nehaniv \& B. Gorayska, Tokyo, IEEE Computer Society. 